Please do not remove this page

RMIT

UNIVERSITY

\title{
Dissociation of prefrontal cortex and nucleus accumbens dopaminergic systems in conditional learning in rats
}

George, D; Jenkins, Trisha; Killcross, Simon

https://researchrepository.rmit.edu.au/esploro/outputs/9921858266001341/filesAndLinks?institution=61RMIT_INST\&index=null

George, D., Jenkins, T., \& Killcross, S. (2011). Dissociation of prefrontal cortex and nucleus accumbens dopaminergic systems in conditional learning in rats. Behavioural Brain Research, 225(1), 47-55.

https://doi.org/10.1016/j.bbr.2011.06.028

Document Version: Accepted Manuscript

Published Version: https://doi.org/10.1016/j.bbr.2011.06.028

Repository homepage: https://researchrepository.rmit.edu.au

(C) 2011 Elsevier B.V.

Downloaded On 2023/04/26 21:18:08 +1000

Please do not remove this page 
For Behavioural Brain Research.

Dissociation of prefrontal cortex and nucleus accumbens dopaminergic systems in conditional learning in rats.

David N. George ${ }^{1}$, Trisha A. Jenkins ${ }^{2}$ and Simon Killcross ${ }^{3}$

${ }^{1}$ Cardiff University, Cardiff, UK; ${ }^{2}$ RMIT University, Melbourne, Australia; ${ }^{3}$ University of New South Wales, Sydney, Australia

Address for correspondence:

David N. George

School of Psychology

Cardiff University

Tower Building

70 Park Place

Cardiff CF10 3AT

United Kingdom

Tel: +44 (0) 2920876286

Fax: +44 (0) 2920874858

Email: GeorgeD@Cardiff.ac.uk

Running head: Dopamine in conditional learning.

Number of pages: 33

Number of figures: 3

Number of tables: 1

Words in manuscript: 7041 (excluding references)

Words in abstract: 249

Words in introduction: 496

Keywords: schizophrenia, microdialysis, HPLC, context, instrumental 


\begin{abstract}
There is converging evidence that the prefrontal and mesolimbic dopaminergic (DAergic) systems are involved in the performance of a variety of tasks that require the use of contextual, or task-setting, information to select an appropriate response from a number of candidate responses. Performance on tasks of this nature are impaired in schizophrenia and in rats exposed to psychotomimetics; impairments that are often attenuated by administration of dopamine (DA) antagonists. Rats were trained on a conditional instrumental discrimination task where auditory cues signalled that responses on one or the other of two levers were reinforced. Animals in a control condition were exposed to a simple discrimination using the same auditory cues and schedules of reinforcement, but with no conditional relationship between the two. Following training, microdialysis probes were implanted unilaterally in either the medial prefrontal cortex (mPFC) or nucleus accumbens (NAc) and samples were collected in free moving animals during a behavioural test session and analyzed using high-performance liquid chromatography. In Experiment 1, we found higher levels of DA in the mPFC of rats performing the conditional discrimination task relative to rats in the simple discrimination control condition. In Experiment 2, rats performing the conditional discrimination showed lower levels of DA in the NAc compared to the control group. These results provide direct evidence that conditional discrimination tasks engage frontal and mesolimbic DAergic systems and are consistent with the proposal that frontal DA is involved in aspects of cognitive control that are known to be impaired in individuals with schizophrenia.
\end{abstract}




\section{Introduction}

One of the core cognitive deficits that have been identified in individuals suffering from schizophrenia is a difficulty in making use of information in the world to direct their responses appropriately (Cohen and Servan-Schreiber, 1992). This deficit is thought to be responsible for impairment to the performance of a number of tasks, such as the Stroop task (Wysocki \& Sweet, 1985), the lexical ambiguity task (Cohen et al., 1988) and the continuous performance task (Barch et al., 2003), each of which relies on the use of tasksetting cues to direct behaviour.

Converging evidence supports the hypothesis that frontal DAergic systems are involved in the performance of such conditional learning tasks. Petrides $(1985,1990,1997)$ trained people on conditional associative learning tasks where they had to learn arbitrary associations between specific stimuli and responses. Patients with frontal lesions demonstrated significantly impaired performance on these tasks. Hofer et al. (2001) reported impaired conditional discrimination learning in individuals with schizophrenia, and Robbins and Sahakian (1983) observed impaired performance on a conditional discrimination task in rats following systemic treatment with D-amphetamine. In a task similar to that employed by Robbins and Sahakian, Dunn et al. (2005) trained rats to make one response in the presence of a tone (e.g. left lever press) and another in the presence of a clicker (e.g., right lever press) for reward. They found that rats' performance on this task was also disrupted by systemic administration of D-amphetamine at doses which did not affect basic sensory, motor, or motivational processes. Furthermore, they showed that pre-treatment with the $D_{1} / D_{2}$ DA receptor antagonist $\alpha$-flupenthixol attenuated the $D$ amphetamine induced impairment. Similarly, Dunn and Killcross (2007b) found that infusion of $\alpha$-flupenthixol into the mPFC attenuates D-amphetamine-induced impairment of this task. 
We sought to provide direct evidence that frontal DAergic systems are involved in the performance of the type of conditional discrimination tasks described above. Using in vivo microdialysis, we measured extracellular DA concentrations in the mPFC (Experiment 1) and the NAc (Experiment 2) of rats performing either a conditional instrumental discrimination or a control simple discrimination. For animals performing the conditional discrimination, two auditory cues signalled which of two candidate responses was reinforced. One auditory cue signalled that left lever presses were reinforced whereas right lever presses were not. The other cue signalled the opposite relationship. Animals in the control group learned one of two simple discriminations where there was no conditional relationship between the cues and the identity of the reinforced response. For some animals, one cue signalled that both responses were reinforced, whereas the other signalled that neither was; for other animals, one response was reinforced in the presence of either cue, whereas the other response was never reinforced. In the conditional and simple discrimination tasks, animals were exposed to the same auditory cues, the same responses and the same schedules of reinforcement, meaning that any differences in DA between the groups can only be attributed to differences in the task demands of conditional and simple discrimination learning.

\section{Materials and Methods}

\section{Subjects.}

A total of 64 male Lister hooded rats (Harlan UK, Bicester, UK) were used in this study, 32 in Experiment 1, and 32 in Experiment 2. Prior to the beginning of each experiment the rats were reduced to $85 \%$ of their age-matched free-feeding weights and were maintained at this level throughout the experiment by being fed a restricted amount after each experimental session. The rats had free access to water in their home cages. They were 
housed in pairs in a light-proof holding room maintained on a 14hr light/ 10hr dark cycle (lights on from 6 am to $8 \mathrm{pm}$ ), at a temperature of $21 \pm 1^{\circ} \mathrm{C}$ and a humidity of $55 \pm 5 \%$. The subjects were tested on successive days, at the same time, during the period that the lights were on in their holding room. At the start of the experiment, one of each housing pair was randomly assigned to the conditional discrimination group, and the other was assigned to the simple discrimination group. All experimental procedures involving animals and their care were performed in accordance with the United Kingdom Animals Scientific Procedures Act (1986) and were subject to Home Office approval (Project License PPL 30/2158).

\section{Surgery.}

Following the eight sessions of training (see below), rats were given free access to food in their home cages. On the next day they were anaesthetized using an isoflurane/oxygen/nitrous oxide mix, their heads were shaved and they were placed in a stereotaxic frame (Kopf Instruments, Tujunga, CA). An incision was made in the scalp, the skull exposed, and a hole above the mPFC (Experiment 1) or NAc (Experiment 2) was drilled out. An MBR-10 guide cannula (Bioanalytical Systems Inc., West Lafayette, IN) was lowered into the brain. For Experiment 1, the guide cannula was aimed at the mPFC: anteroposterior $(A P)+3.0 \mathrm{~mm}$, mediolateral $(\mathrm{ML})-0.6 \mathrm{~mm}$, dorsoventral (DV) $-3 \mathrm{~mm}$. In Experiment 2 the guide cannula was aimed at the NAc (AP +1.7, ML -1.7, DV -6.4). The guide cannula was fixed to the skull using dental cement (Simplex Rapid Liquid and Powder; Associated Dental Products Limited, Swindon, UK) and three fixing screws located on different bone plates. A split screw was embedded in the dental cement to allow attachment to a M115 head block tether (Instech Laboratories Inc., Plymouth Meeting, PA). After recovery from anaesthesia, each rat was returned to its home cage with free 
access to food and water. After a minimum of 1 week of postoperative recovery, rats were again gradually reduced to $85 \%$ of age-matched free-feeding weights.

Histology.

At the completion of the test session, rats were given a lethal overdose of sodiumpentobarbitone. Brains were excised and post-fixed in formal saline $(10 \% \mathrm{w} / \mathrm{v})$. Before slicing they were transferred to a $25 \%$ sucrose solution in which they remained for $24 \mathrm{hr}$. Coronal slices $(40 \mu \mathrm{m})$ were cut using a cryostat (Leica Microsystems GmbH, Wetzlar, Germany) and were mounted onto gelatin-coated slides. These were dried at room temperature for $24 \mathrm{~h}$ then oven dried at $40^{\circ} \mathrm{C}$ before staining with Cresyl violet, followed by the addition of a coverslip in DPX. The precise placement of the microdialysis probes was verified using a light microscope and the brain atlas of Paxinos and Watson (1998).

\section{Behavioral apparatus.}

Training and testing took place in two operant conditioning chambers measuring $30.5 \mathrm{~cm}$ wide $\times 24.1 \mathrm{~cm}$ deep $\times 29 \mathrm{~cm}$ high (Med Associates Inc., St. Albans, VT). The left and right walls of the chamber were aluminium, whereas the rear wall, ceiling, and a door which served as the front wall were made of clear Plexiglass. The grid floor of the chamber consisted of 19 steel rods $4.8 \mathrm{~mm}$ in diameter, spaced $1.6 \mathrm{~cm}$ apart. There was a $5.1 \mathrm{~cm}$ diameter hole in the centre of the ceiling with a $6.4 \mathrm{~mm}$ access slot cut from the hole to the front edge. An Instech MCLA 15-cm counter-balanced lever arm was attached on top of the ceiling so that a liquid swivel could be suspended directly above the hole. The chambers were dimly illuminated by a 3-watt house-light located at the top centre of the rear wall and oriented towards the ceiling. Food pellets (45mg; TestDiet, Richmond, IN) 
were delivered into a recessed magazine located in the right hand wall of each chamber. The entrance to the magazine measured $5.1 \mathrm{~cm} \times 8 \mathrm{~cm}$ and allowed the animals to retrieve food with head-tethers attached. Two flat-panel retractable levers were fitted to the left and right of the food magazine. Auditory stimuli consisted of a $2 \mathrm{kHz}$ tone delivered from an $8-\Omega$ speaker located near the ceiling in the left wall and a $10 \mathrm{~Hz}$ train of clicks generated by operating a mechanical relay mounted next to the speaker. Events in the chambers were controlled, and responses recorded, by an IBM compatible microcomputer running the MED-PC IV software (Med Associates Inc.).

Neurochemical apparatus.

On the test day, an MBR-2-10 microdialysis probe (Bioanalytical Systems Inc., West Lafayette, IN) with $2 \mathrm{~mm}$ exposed length of dialysis membrane was inserted into the implanted guide cannula and connected using FEP tubing (inner diameter $0.12 \mathrm{~mm}$; CMA Microdialysis, Solna, Sweden) to an Instech (375/D/22QM) quartz-lined dual-channel swivel and artificial CSF (147 mM NaCl, $2.7 \mathrm{mM} \mathrm{KCl}, 1.2 \mathrm{mM} \mathrm{CaCl}, 0.85 \mathrm{mM} \mathrm{MgCl}$; CMA Microdialysis) was perfused at a constant rate of $2 \mu \mathrm{L} / \mathrm{min}$. Dialysis samples were collected every 10 minutes by a HoneyComb refrigerated fraction collector (Bioanalytical Systems Inc.) into $10 \mu \mathrm{L}$ of $0.1 \mathrm{M}$ perchloric acid (PCA).

At the end of the test session, all dialysis samples were analyzed using a small-bore highperformance liquid chromatography (HPLC) system consisting of a Dionex P680A isocratic pump, (Dionex Corporation, Sunnyvale, CA), a Dionex ASI-100T automatic sample injector with a $50 \mu \mathrm{L}$ loop, a Waters Spherisorb column (5 $\mu \mathrm{m}$ particles; $4.6 \mathrm{~mm}$ x $150 \mathrm{~mm}$; Waters Corporation, Milford, MA) mounted in a Dionex STH-585 column oven maintained at $30^{\circ} \mathrm{C}$, and an ESA Coulochem II electrochemical detector (ESA Biosciences Inc., Chelmsford, MA) with ESA 5020 guard cell (operated at $350 \mathrm{mV}$ ) and ESA 5014B microdialysis cell 
(electrode 1 operated at $100 \mathrm{mV}$, electrode 2 at $250 \mathrm{mV}$ ). The HPLC system was controlled online, and data were collected, by an IBM compatible microcomputer running Dionex's Chromeleon software.

The mobile phase consisted of HiPerSolv (BDH, Poole, UK) water with 20 mM tri-sodium citrate, $44 \mathrm{mM}$ sodium dihydrogen orthophosphate, $2 \mathrm{mM}$ heptane sulphonic acid, $100 \mu \mathrm{M}$ EDTA and 14\% (v/v) HiPerSolv methanol. The acidity of the mobile phase was adjusted to $\mathrm{pH} 3.0$ by the addition of orthophosphoric acid. The detection threshold of the system (2:1 ratio of signal to background noise) for DA was $0.5 \mathrm{pg}$ injected onto the column.

\section{Behavioural procedure}

Magazine and lever-press training. All rats were given a single 30-minute session of magazine training in which a single food pellet was delivered according to a random-time, 60-s schedule. On each of the following two days the animals received a 48-minute session of lever press training. During each of these sessions each lever was presented 12 times in a pseudo-random order. Each presentation lasted for $60 \mathrm{~s}$ and responding on each lever was reinforced according to a random-interval, 15-s schedule (each second there was a one in 15 chance that food would become available after which the first response was rewarded, hence food was delivered on average once every $15 \mathrm{~s}$ ). The mean interval between lever presentations was $60 \mathrm{~s}$ (range 30-90 s).

Discrimination training. The design of the experiment is shown in Table 1. On each trial, two response levers were inserted into the conditioning chamber and one of two auditory cues (a tone and a clicker) was presented. For half of the animals in the conditional discrimination group, responding on the left lever was reinforced in the presence of the tone, but not in the presence of the clicker; whereas responding on the right lever was 
reinforced in the presence of the clicker, but not in the presence of the tone. For the remaining animals in this group these contingencies were reversed. For the animals in simple discrimination group, reinforcement was signalled by either the auditory cue or by position. Hence, there were four counterbalancing conditions for the simple discrimination group: i) responding on the left lever was reinforced whereas responding on the right lever was not; ii) responding on the right lever was reinforced whereas responding on the left lever was not; iii) responding on either lever was reinforced in the presence of the tone, but not in the presence of the clicker; iv) responding on either lever was reinforced in the presence of the clicker, but not in the presence of the tone.

$<$ Table 1 about here $>$

Over the eight days following magazine and lever-press training, all animals received presurgery discrimination training. On each trial, both levers were extended into the chamber and one of the two auditory discriminative stimuli $\left(S_{D}\right)$ was presented. The trials each lasted for $60 \mathrm{~s}$ at the end of which the levers were withdrawn and the $S_{D}$ terminated. In each session the animals received 12 trials with each $S_{D}$, presented in a pseudo-random order with the constraint that no more than two trials with the same $S_{D}$ were presented in succession. The mean interval between the end of one trial and the beginning of the next one was $60 \mathrm{~s}$ (range 30-90 s) and each session again lasted for 48 minutes. The chamber was illuminated by the house-light for the duration of the session. Responses on the levers were, where appropriate, reinforced according to a random-interval, 15-s schedule. To provide an index of conditioned responding uncontaminated by reinforcement, responding during the first $10 \mathrm{~s}$ of a trial was never reinforced. All analyses of behavioural performance are based on the data from this $10 \mathrm{~s}$ period.

Following recovery from surgery, animals were given a single retraining session the details of which were the same as for the discrimination training sessions. On the following day, 
microdialysis probes were inserted and rats were placed in the operant chamber and allowed to explore for 60 minutes before the collection of microdialysis samples commenced. Following a further 60 minutes, during which baseline samples were collected, the house-light was illuminated and the test session began. Details of the test session were the same as for the discrimination training session with the exception that the test session consisted of 30 trials (15 with each auditory cue) and lasted for 60 minutes.

A random-interval reinforcement schedule was employed to maintain consistency with the pharmacological studies which had revealed a link between conditional discrimination performance and DA receptor agonism (Dunn et al., 2005; Dunn \& Killcross, 2007a, b). Although random- and variable-interval schedules are less commonly employed in microdialysis studies than fixed-ratio schedules, their use in not unknown (e.g., Ahn \& Phillips, 2007; Nomura et al., 2004), and NAc DA release in response to lever-pressing for food reward under these different schedules is known not to differ (Sokolowski et al., 1998).

All animals received presentations of the same two stimuli, a tone and a clicker, and had access to both response levers on each trial. For rats learning the conditional discrimination, and those in two of the counterbalancing conditions for the simple discrimination, responding on each of the levers was reinforced on half of the trials. For animals in the remaining counterbalancing conditions for the simple discrimination, responding on one lever was reinforced on all trials, whereas responding on the other lever was never reinforced. Because the schedules of reinforcement on the two levers were independent of each other, this meant that all animals had the same opportunity to earn reward over the course of a session. Hence, the only difference in the treatment of animals learning the conditional or simple discriminations was that in the former case there 
was a conditional relationship between the auditory cues and the identity of the reinforced lever; whereas in the latter case either the cue, or the identity of the lever alone signalled reinforcement.

In the current study guide cannulae were implanted approximately a week prior to testing, however the microdialysis probes were inserted one hour prior to commencing collection of baseline samples. It is important to note that, under these circumstances, there is evidence that a significant proportion of sampled DA may not derive from exocytotic release. For example, Santiago \& Westerink (1990) found that when samples were taken from a probe 2 hours after it was inserted into a chronically implanted guide cannula, only $40 \%$ of DA released in the striatum was sensitive to blockade of neuronal activity by infusion of the $\mathrm{Na}+$-channel blocker tetrodotoxin. Importantly, there are two reasons why these considerations do not affect the interpretation of our results. First, a considerable proportion of DA sampled under these conditions remains sensitive to tetrodotoxin. Second, with the exception of the type of discrimination that an animal was trained on (conditional vs. simple), each pair of rats was treated identically in all respects including time of surgery and probe insertion. Any consistent difference in DA efflux between animals in the two groups must, therefore, be a consequence of different task demands of the two discriminations.

\section{Statistical methods}

Throughout training and testing the subjects were treated as matched pairs which were trained at the same time each day, received surgery on the same day, were tested at the same time, and whose microdialysis samples were processed by HPLC in the same batch. Within each pair, one rat belonged to the conditional discrimination group, and the other belonged to the simple discrimination group. Because rats were treated as matched pairs 
in this way, all data were analyzed using within-subject (related samples) statistical methods.

Response rates during the initial $10 \mathrm{~s}$ of each $\mathrm{S}_{\mathrm{D}}$ presentation were analyzed to yield a measure of conditioned responding that was not contaminated by the delivery of reinforcement. In order to simplify analysis of behavioural data, rates of responding on the two levers during the two $S_{D} S$ were combined to give an overall measure of task performance. Responses on a lever that was associated with reward in the presence of a given $S_{D}$ were labelled 'correct', whereas responses on a lever that was not associated with reward in the presence of that $S_{D}$ were labelled 'incorrect'. For animals in the simple discrimination group where responses on one lever were reinforced in the presence of either $S_{D}$, but responses on the other lever were never reinforced, responses on the reinforced lever were correct whereas responses on the non-reinforced lever were incorrect. For the remaining simple discrimination rats for which responding on either lever was reinforced in the presence of one $S_{D}$, but not in the presence of the other $S_{D}$, responses made during presentations of the former $S_{D}$ were correct and responses made in the presence of the latter $S_{D}$ were incorrect.

Neurochemical concentrations were expressed as percentages of baseline levels. The average concentrations of the six samples preceding the start of the test session was used as the basal level for each animal. Because DA concentrations in all samples were evaluated with respect to these six samples, they were not themselves included in further analysis. There were, however, no differences in baseline levels of DA between animals performing the conditional and simple discriminations, either overall or for any of the six individual sampling periods. 
Data were analysed using Student's $t$-test and analysis of variance (ANOVA). If an interaction was significant $(p<0.05)$ simple effects were examined and, where appropriate, post-hoc pair-wise comparisons were made using the Tukey-Kramer test.

\section{Results}

\section{Experiment 1}

Histology. The aim of this experiment was to measure performance related changes in extracellular DA levels in the mPFC. No animals were excluded from analysis on the basis of probe placement, but two pairs of animals were excluded due to HPLC or microdialysis failure. Probe placements for the remaining animals are shown in Figure $1 A(n=28)$.

\section{$<$ Figure 1 about here>}

Behavioural results. Panel A of Figure 2 shows the mean rates of correct and incorrect responding for rats learning the conditional or simple discrimination in Experiment 1. By the end of training, animals in both groups had successful acquired the discrimination, making more correct than incorrect responses during presentations of both $S_{D} S$. On the retraining session administered the day before the test session, performance of the two discriminations was equivalent. A two-way ANOVA with the within-subject factors of discrimination (conditional vs. simple) and lever (correct vs. incorrect) confirmed this observation. This ANOVA revealed an effect of lever $\left(F_{1,13}=89.28 ; P<0.001\right)$, but no effect of discrimination $\left(F_{1,13}=2.62, P=0.13\right)$, and no interaction involving these factors $(F<1)$

During the test session of Experiment 1, animals continued to make more correct than incorrect lever-press responses. A two-way ANOVA with the within-subject factors of discrimination and lever confirmed that there was a significant effect of lever $\left(F_{1,13}=\right.$ 
64.72; $P=0.001)$, but no effect of discrimination $\left(F_{1,13}=1.78, P=0.21\right)$ and no significant interaction $\left(F_{1,13}=2.37, P=0.15\right)$.

Since the expectation of food reward increases DA in both mPFC (Merali et al., 2004) and NAc (Phillips et al., 1993; Bassareo and Di Chiara, 1999), and feeding increases levels of DA in the NAc of food-deprived animals (e.g., Heffner et al., 1980) it is important to show that there was no difference in the number of pellets earned by animals learning the two discriminations either by the end of training, or during the behavioral test session. During the post-operative retraining session, the mean number of pellets earned was $65.7 \pm 7.0$ for the conditional discrimination group and $48.2 \pm 9.0$ for the simple discrimination group. During the behavioural test session of, animals in the conditional discrimination group earned an average of $68.6 \pm 9.5$ pellets, whereas those in the simple discrimination group earned an average of $61.1 \pm 9.4$ pellets. A two-way ANOVA with the within-subject factors of discrimination (conditional vs. simple) and session (retraining vs. test) revealed no effect of discrimination $\left(F_{1,13}=1.71, P=0.22\right)$ or session $\left(F_{1,13}=2.50, P=0.14\right)$ and no interaction between these factors $\left(F_{1,13}=1.13, P=0.31\right)$.

Neurochemical results. Baseline levels of DA in the dialystate $( \pm 1$ S.E.M.) were $0.12 \pm$ 0.02 pmol per sample for the mPFC. A related-samples $t$-test revealed no differences in the basal levels of DA between the two groups (conditional $=0.11 \mathrm{pmol}$, simple $=0.13$ pmol $[t<1])$.

There was a greater increase in DA efflux in the mPFC in animals performing the conditional discrimination than in those performing the simple discrimination (Figure 3A). This observation was confirmed by a two-way ANOVA with the within-subject factors of discrimination (conditional vs. simple) and time (the 12 samples collected during the test session and recovery period), which found an effect of discrimination $\left(F_{1,13}=17.66 ; P=\right.$ 0.001). Although the difference between the groups appeared to be greater during the 
recovery period than during the behavioral test session, due largely to much higher levels of DA efflux in animals in conditional discrimination group during the recovery period, this apparent effect was not reflected by the findings of the ANOVA. Although there was an effect of time $\left(F_{11,143}=3.91 ; P<0.001\right)$, there was no interaction of the two factors $\left(F_{11}\right.$, $143=1.47)$.

The failure to find a significant interaction of discrimination and time could be a consequence of the degree of variability of DA levels in animals performing the conditional discrimination task over the six samples collected during the test session. To test for this possibility, we analyzed the mean levels of DA over the six samples collected during the test session and the six samples collected during the post-test recovery period. Average levels of DA efflux for animals in the conditional discrimination group were $106.5 \%$ and $146.4 \%$ of basal levels during the test and recovery periods, respectively. The corresponding levels for animals in simple discrimination group were $94.9 \%$ and $107.7 \%$ of basal levels. A two-way ANOVA with the within-subject factors of discrimination and sample period (test vs. recovery) revealed significant effects of discrimination $\left(F_{1,13}=\right.$ 17.66; $P=0.001)$ and period $\left(F_{1,13}=9.35 ; P=0.009\right)$ and a significant interaction of these factors $\left(F_{1,13}=9.69 ; P=0.008\right)$. Subsequent simple effects analyses revealed a significant effect of period for animals performing the conditional discrimination $\left(F_{1,26}=\right.$ 17.05; $P<0.001)$, but not for those performing the simple discrimination $\left(F_{1,26}=1.79, P=\right.$ 0.19), and a significant effect of discrimination during the recovery period $\left(F_{1,26}=27.35 ; P\right.$ $<0.001)$, but not during the test period $\left(F_{1,26}=2.48, P=0.13\right)$. Hence, animals performing the conditional discrimination showed significantly greater mPFC DA efflux during the recovery period than during the test session and significantly greater DA efflux during the recovery period than animals performing the simple discrimination task. One-sample $t$ tests revealed that DA efflux was significantly greater than baseline levels for animals performing the conditional discrimination during the recovery period $\left(t_{13}=4.62, P<0.001\right.$, 
Bonferroni corrected alpha $=0.0125)$, but not during the test session $(t<1)$. DA efflux did not differ from baseline levels during either the test session or the recovery period for animals performing the simple discrimination $(t s<1)$.

$<$ Figures 2 and 3 about here $>$

\section{Experiment 2}

Histology. The aim of this experiment was to measure extracellular DA levels in the NAc during performance of conditional and simple discrimination task. When the location of the microdialysis probe for either animal in a pair fell outside the NAc, data from both animals were removed from the experiment. Consequently, one pair of rats was excluded. Two further pairs of animals were excluded as a result of failure of microdialysis probes or the HPLC system. Figure 1B depicts the placement of probes within the NAc for $(n=26)$.

Behavioural results. All animals in both the conditional and simple discrimination groups learned to respond more on the correct lever than on the incorrect lever. Panel B of Figure 2 shows the mean rates of correct and incorrect responding for rats learning the two discriminations. A two-way ANOVA with the within-subject factors of discrimination (conditional vs. simple) and lever (correct vs. incorrect) revealed that rats made significantly more correct than incorrect responses during the retraining session $\left(F_{1,12}=\right.$ 64.43; $P<0.001)$, but that there was no effect of discrimination $\left(F_{1,12}=2.17, P=0.17\right)$, and no interaction of lever and discrimination $(F<1)$. A second two-way ANOVA with the same factors was performed on the data from the test session. This analysis also revealed a significant effect of lever $\left(F_{1,12}=18.20 ; P=0.001\right)$, but no effect of discrimination and no interaction of these factors $(F s<1)$.

During the post-operative retaining session, the mean number of pellets earned by animals in the conditional discrimination group was $56.1 \pm 8.7$ and by animals in the simple 
discrimination group was $49.6 \pm 7.1$. During the test session, the corresponding numbers were $45.1 \pm 7.5$ and $42.5 \pm 7.5$. A two-way ANOVA with the within-subject factors of discrimination (conditional vs. simple) and session (retraining vs. test) revealed no effect of discrimination $(F<1)$ or session $\left(F_{1,12}=2.65, P=0.13\right)$ and no interaction between these factors $(F<1)$.

Neurochemical results. Baseline levels of DA in the dialystate $( \pm 1$ S.E.M.) were $0.19 \pm$ 0.02 pmol per sample for the NAc. A related-samples $t$-test revealed no differences in the basal levels of DA between the two groups in (conditional $=0.19 \mathrm{pmol}$, simple $=0.19 \mathrm{pmol}$ $[t<1])$

A greater increase in DA efflux in the NAc was found in animals performing the simple discrimination than in those performing the conditional discrimination (Figure 3B). There was a general increase in the levels of DA efflux from the start of the behavioural test session which continued throughout the 60-min recovery period. A two-way ANOVA with the within-subject factors of discrimination and time found effects of discrimination $\left(F_{1,12}=\right.$ 9.03; $P=0.011)$ and time $\left(F_{11,132}=6.06 ; P<0.001\right)$. The interaction of these factors did not reach significance $(F<1)$. When DA levels were averaged over the six samples from the test period and the six sample from the recovery period, a similar pattern was observed. A two-way ANOVA with the within-subject factors of discrimination and sample period (test vs. recovery) revealed significant effects of discrimination $\left(F_{1,12}=9.03 ; P=\right.$ $0.011)$ and period $\left(F_{1,13}=10.25 ; P=0.008\right)$ and no significant interaction of these factors $(F<1)$. Finally, one-sample $t$-tests revealed that DA efflux did not differ significantly from baseline levels for animals performing the conditioning discrimination during the test period $\left(t_{12}=2.69, P=0.019\right.$, Bonferroni corrected alpha $\left.=0.0125\right)$, but was significantly greater than baseline for these animals during the recovery period $\left(t_{12}=3.95, P=0.002\right)$, and for 
the animals performing the simple discrimination during both the test $\left(t_{12}=8.15, P<\right.$ $0.001)$ and recovery $\left(t_{12}=4.77, P<0.001\right)$ periods.

\section{Discussion}

In each of two experiments we found differential changes in levels of extracellular DA in animals that had learned a conditional or a simple operant discrimination task. In Experiment 1, we observed higher levels of extracellular DA in the mPFC of animals following performance of a conditional instrumental discrimination, than following performance a simple instrumental discrimination. In Experiment 2 we found the converse pattern in the NAc; there was greater DA release in animals performing the simple discrimination than in those performing the conditional discrimination. These findings are consistent with the hypothesis that the mPFC and mesolimbic DAergic systems are involved in the performance of tasks that involve making use of task-setting cues to direct behaviour. More specifically, they add to the growing body of evidence that the mPFC is involved in conditional discrimination learning (Bussey et al., 2001, 2002; Cadoret et al., 2001; Dunn \& Killcross, 2007b; Xiang \& Brown, 2007), and provide direct evidence that the frontal DAergic system is engaged by conditional discrimination tasks.

These results are most likely to reflect changes in DA efflux as a consequence of performance of the different discrimination tasks and not due to non-specific influences of the training or test procedures. There is evidence both that eating increases DA levels in the NAc (Hernandez and Hoebel, 1988) and that lever-pressing for a food reward increases DA in the mPFC (Hernandez and Hoebel, 1990; Izaki et al., 1998). Because there were no differences in either the number of reinforcers earned by animals in the conditional discrimination group and the simple discrimination group, nor in their rates of responding, however, neither of these factors nor their interaction can be responsible for 
the differences in levels of extracellular DA observed between the two groups. Feenstra (2007, see also Neill et al., 2002; Sokolowski et al., 1998) has suggested that changes in mPFC DA efflux are not the result of either consumatory behaviour or lever pressing, but reflect the task demands of appetitive instrumental tasks; specifically, the effort required to perform the correct response. Unsurprisingly, considering that we found no group difference in response or reinforcement rates, we found no differences in effort either. For example, the number of responses made per reinforcer by animals learning the two discriminations (the ratio of response rate to reinforcement rate during the test session) did not differ between the two discriminations in either Experiment 1 or Experiment 2 (ts $<1$ ), regardless of whether only correct, or both correct and incorrect, responses were used to calculate response rate. Hence, any difference in the effort required to make a correct response in the conditional and simple discrimination tasks must reflect the task-setting requirement of the former task, where rats had to integrate information about the auditory cues and the identities of the levers. Indeed, it seems that it is specifically the conditional nature of the task which is responsible for higher levels of mPFC DA. On several occasions Killcross and his colleagues have employed a Pavlovian to instrumental transfer (PIT) test as a control for performance in a conditional instrumental learning task. PIT has been used to show that administration of systemic d-amphetamine (Dunn et al., 2005) or phencyclidine (Dunn and Killcross, 2007a) do not disrupt rats' ability to discriminate between auditory cues or levers, nor more importantly their ability to integrate information about cues and levers. Treated rats still demonstrated a selective increase in responding on a reinforced lever during the presentation of a reinforced Pavlovian cue, despite using doses that impair conditional discrimination performance.

The complimentary patterns of mPFC and NAc DA efflux in animals performing the two discrimination tasks is consistent with what is known about the circuitry of the mPFC and mesolimbic DAergic systems. Using a triple labelling method, Carr and Sesack (2000; 
Sesack \& Carr, 2002) identified the projection targets of DA and GABA neurons in the ventral tegmental area which receive input from PFC pyramidal neurons. They found that PFC afferents target mesoprefrontal DA, but not GABA, neurons; and mesoaccumbens GABA, but not DA neurons, as well as GABA interneurons that inhibit mesoaccumbens DA cells. This pattern of connectivity means that PFC activity will result in an increased level of PFC DA efflux and an accompanying decrease in NAc DA release. Direct evidence for this reciprocal relationship between MPFC activity and NAC DA release has been provided by Jackson et al. (2001) who found that electrophysiological stimulation of the mPFC at frequencies matching mPFC neuron firing rate in rats performing cognitive tasks resulted in a decrease in NAc DA levels (see also Wilkinson et al., 1998). Hence, greater increases in mPFC DA in animals performing the conditional discrimination may be responsible for the observed reduction in NAc DA relative to animals in our simple discrimination group.

One noteworthy aspect of our data is that DA levels remain elevated for some time following the end of the test session. This is true in the NAc of animals performing either the simple or conditional discrimination task, and in the mPFC of animals performing the conditional task. During the recovery period, all animals remained in the darkened conditioning chamber in the absence of discriminative cues or response levers; there were no observable differences in the behaviour of rats in the conditional and simple discrimination groups. Although these results seem to suggest that DA efflux is not necessarily directly linked to the overt behaviour expressed by our rats, they are consistent with previous research. For example, Hernandez and Hoebel (1988; see also Louilot et al., 1986) observed elevated levels of DA in the NAc of rats pressing a bar for food in the presence of a signal for reward. The increase in DA endured for some considerable time after the signal terminated and food was no longer available, whilst the rats seldom pressed the lever when the signal was not present. These data, and ours, are consistent with the idea that environmental cues may trigger an increase in DA efflux that serves to 
modulate complex behaviour and that this modulation persists longer than the behaviour. It is likely that the greater increase in DA levels in the NAc of animals in simple discrimination group (Fig 3A) reflects the down-regulation of NAc DA in conditional discrimination group due to increased mPFC activity (Fig 3B, Deutch et al., 1990; King et al., 1997). Stefani and Moghaddam (2006; see also Phillips et al., 2004; Rossetti \& Carboni, 2005) also found that elevation of DA efflux in the mPFC persisted following training. Over two sessions, Stefani \& Moghaddam (2006) trained animals on a plus-maze set-shifting task. In the first session they were required to learn a discrimination between two responses (turn left or turn right) on the basis of either the brightness or texture of the maze arms. Following a two hour interval, the rats were returned to the maze and required to learn the alternate discrimination. mPFC DA levels were elevated above baseline during both training sessions, and remained elevated for at least 40 minutes following training. The authors suggested that this sustained change in DA activity may be important for working memory, memory consolidation, or other higher-order processes dependent on the organization of behaviour.

These proposals are in line with work suggesting a role for PFC DA in cognitive control (Braver and Cohen, 2000; Braver et al., 1999; Goldman-Rakic, 1991). In a formal model based on the role of contextual, or task-setting, cues in cognitive control (Cohen et al., 1990), Braver and colleagues have suggested that DA plays a role in modulation of PFCdependent memory systems that activate and maintain contextual cues that are needed for the task solution. Moreover, the activation of these cues persists beyond their period of presentation. They propose that optimal levels of PFC DA are required for efficient regulation of appropriate task-set, and further suggest dissociable roles for tonic and phasic DA in the maintenance and production of contextual control (see also Braver and Barch, 2006). The findings we present here are in line with the spirit (if perhaps not all the details) of Cohen et al.'s (1990) model. With its low temporal resolution, microdialysis is 
suited to measuring relatively slow fluctuations in tonic levels of extracellular DA. In contrast, the sub-second resolution of fast scan cyclic voltammetry has recently allowed the measurement of phasic changes in NAc DA efflux in response to both Pavlovian reward predicting cues (e.g., Struber et al., 2008; Sunsay \& Rebec, 2008) and instrumental $S_{D} S$ (Jones et al., 2010), which microdialysis is insensitive to. Hence, it is unlikely that the changes in DA release which we observed are directly related to animals' response production. Rather, we argue that tonic changes in DA in mPFC (and corollary changes in NAc) are needed to allow PFC systems to adopt and maintain appropriate task-setting contextual cues as controllers of behaviour, and that these levels of DA (and hence capacity for cognitive control) are maintained beyond the period of cue presentation.

In concert with this work, Cohen \& Servan-Schreiber (1992) identified a core cognitive deficit in schizophrenia as a disturbance in this internal representation of context. Patients with schizophrenia display an impaired ability to construct, maintain, and manipulate representations of contextual information required to direct appropriate responses. A failure to properly process such task-setting information may be responsible for poor performance on a wide range of conditional tasks including the Stroop task (Wysocki \& Sweet, 1985), the lexical ambiguity task (Cohen et al., 1988), and the continuous performance task (Cornblatt et al., 1989). Dunn et al. (2005) noted the similarity of the CPT-AX task (e.g., Javitt et al., 2000; Umbricht et al., 2000) and the conditional instrumental discrimination procedure used in our, and their, experiments. In the CPT-AX, a participant must make a response to the letter $X$ if preceded by the letter $A$, and to withhold responding when $\mathrm{X}$ is preceded by $\mathrm{B}$; responses to $\mathrm{X}$ are conditional upon the preceding cue. In our conditional instrumental discrimination task, rats were reinforced for responding to the left-lever $(X)$ in the presence of one cue $(A)$, but not the other $(B)$. Furthermore, there is some evidence that patients with schizophrenia are impaired on 
learning tasks that are more directly equivalent to our conditional instrumental discrimination. Hofer et al. (2001) found that patients with schizophrenia showed impaired acquisition of a differential conditioned eyeblink response relative to healthy controls when a tone was followed by an aversive puff of air to the eye if it was preceded by a green light, but not if it was preceded by a red light. Using a conditional associative learning task in which participants were required to pair each of four stimulus cards with different responses, Gold et al. (2000) observed a similar deficit in learning by patients with schizophrenia.

Considerable effort has been devoted to investigating the possible relationship between schizophrenia and dysfunction of PFC and striatal DA systems. On the one hand, an increase in $\mathrm{DA} \mathrm{D}_{2}$ receptor density has been observed in individuals with schizophrenia both in postmorten studies (e.g., Cross et al., 1987) and using neuroimaging of living patients (Wenberger \& Laruelle, 2001); while DA $\mathrm{D}_{2}$ receptor antagonists are therapeutically effective (Carlsson, 1974). On the other hand, core cognitive deficits observed in schizophrenia have been linked with reduced prefrontal activity (Weinberger et al., 1994). This, and other, evidence has lead to the proposal that schizophrenia is associated with elevated striatal, and reduced prefrontal DA activity (Guillin et al., 2007). It is important, therefore, to note that atypical antipsychotics increase PFC DA relative to NAc (Melis et al., 1999; Youngren et al., 1999) and that typical antipsychotic have the opposite effect (Moghaddam \& Bunney, 1990). Consistent with these changes in PFC and striatal DA, atypical antipsychotics produce a greater improvement in cognitive performance than do typical antipsychotics (Guilera et al., 2009). Furthermore, using the same conditional discrimination task reported here, Dunn \& Killcross (2006a, b; 2007a) found that the disruptive effects of phencyclidine and D-amphetamine on performance was attenuated by atypical, but not typical, antipsychotics in rats. 
In summary, following the work of Dunn and Killcross (2007b) demonstrating a role for prefrontal dopaminergic systems in performance of conditional tasks, and previous work demonstrating the utility of conditional task performance as an assay of pharmacological animals models of schizophrenia (Dunn and Killcross, 2007a), we have now shown that performance of conditional tasks produces changes in DA levels in cortical and subcortical regions thought to be key to the deficits observed in schizophrenia. These results reflect the putative role of DA in neural network models of cognitive control, and observed deficits in related tasks in patients with schizophrenia. 


\section{Acknowledgements}

This work was supported by a Royal Society University Research Fellowship awarded to DNG and a NARSAD Zarrow Young Investigator Award to SK. Preparation of this manuscript was supported by NH\&MRC Grant ref 568872 to SK. 


\section{References}

Ahn, S., \& Phillips, A. G. (2007). Dopamine efflux in the nucleus accumbens during withinsession extinction, outcome-dependent, and habit-based instrumental responding for food reward. Psychopharmacology, 191, 641-651.

Barch, D. M., Carter, C. S., MacDonald, A. W., Braver, T. S., \& Cohen, J. D. (2003). Context-processing deficits in schizophrenia: diagnosis, specificity, 4-week course, and relationships to clinical symptoms. J. Abnorm. Psychol., 112, 132-143.

Braver, T. S., \& Barch, D. M. (2006). Extracting core components of cognitive control. Trends Cogn. Sci., 10, 529-532.

Braver, T. S., \& Cohen, J. D. (2000). On the control of control: The role of dopamine in regulating prefrontal function and working memory. In S. Monsell \& J. Driver (Eds.), Attention and Performance XVIII; Control of cognitive processes (pp. 713-737). Cambridge, MA: MIT Press.

Braver, T. S., Barch, D. M., \& Cohen, J. D. (1999). Cognition and control in schizophrenia: A computational model of dopamine and prefrontal function. Biol. Psychiat., 46, 312-328.

Bassareo, V., \& Di Chiara, G. (1999). Modulation of feeding-induced activation of mesolimbic dopamine transmission by appetitive stimuli and its relation to motivational state. Eur. J. Neurosci., 11, 4389-4397.

Bussey, T. J., Wise, S. P., \& Murray, E. A. (2001). The role of ventral and orbital prefrontal cortex in conditional visuomotor learning and strategy use in rhesus monkeys (Macaca mulatta). Behav. Neurosci., 115, 971-982.

Bussey, T. J., Wise, S. P., \& Murray, E. A. (2002). Interaction of ventral and orbital prefrontal cortex with inferotemporal cortex in conditional visuomotor learning. Behav. Neurosci., 116, 703-715.

Cadoret, G., Pike, G. B., Petrides, M. (2001). Selective activation of the ventrolateral prefrontal cortex in the human brain during active retrieval processing. Eur. J. Neurosci., 14, 1164-1170. 
Carlsson, A. (1974). Antipsychotic drugs and catecholamine synapses. J. Psychiat. Res., $11,57-64$.

Carr, D. B., \& Sesack, S. R. (2000). Projections from the rat prefrontal cortex to the ventral tagmental area: Target specificity in the synapse associations with mesoaccumbens and mesocortical neurons. J. Neurosci., 20, 3864-3873.

Cohen, J. D., Dunbar, K., \& McClelland, J. L. (1990). On the control of automatic processes - a parallel distributed-processing account of the Stroop effect. Psychol. Rev., 97, 332-361.

Cohen, J. D., Semple, W. E., Gross, M., Mordahl, T. E., Holcomb, H. H., Dowling, M. S., \& Pickar, D. (1988). The effect of neuroleptics on dysfunction in a prefrontal substrate of sustained attention in schizophrenia. Life Sci., 43, 1141-1150.

Cohen, J. D., \& Servan-Schreiber, D. (1992). Context, cortex, and dopamine: a connectionist approach to behavior and biology in schizophrenia. Psychol. Rev., 99, 45-77.

Cornblatt, B. A., Lezenweger, M. F., \& Erlenmeyer-Kimling, L. (1989). The continuous performance test, identical pairs version: II. Contrasting attentional profiles in schizophrenic and depressed patients. Psychiat. Res., 29, 65-85.

Cross, A. J., Crow, T. J., Ferrier, I. N., Johnstone, E. C., McCreadie, R. M., Owen, F., Owens, D. G., \& Poulter, M. (1983). Dopamine receptor changes in schizophrenia in relation to the disease process and movement disorder. J. Neural. Transm. Suppl., 18, 265-272.

Deutch, A. Y., Clark, W. A., \& Roth, R. H. (1990). Prefrontal cortical dopamine depletion enhances the responsiveness of mesolimbic dopamine neurons to stress. Brain Res., 521, 311-315.

Dunn, M. J., Futter, D., Bonardi, C., \& Killcross, S. (2005). Attenuation of d-amphetamineinduced disruption of conditional discrimination performance by a-flupenthixol. Psychopharmacology, 177, 296-306.

Dunn, M. J., \& Killcross, S. (2006a). Clozapine but not haloperidol treatment reverses subchronic phencyclidine-induced disruption of conditional discrimination performance. Behav. Brain. Res., 175, 271-277. 
Dunn, M. J., \& Killcross, S. (2006b). Differential attenuation of $d$-amphetamine-induced disruption of conditional discrimination performance by dopamine and serotonin antagonists. Psychopharmacology, 188, 183-192.

Dunn, M. J. \& Killcross, A. S. (2007a). Clozapine, SCH 23390 and a-Flupenthixol but not Haloperidol attenuate phencyclidine-induced disruption of conditional discrimination performance Psychopharmacology, 190, 403-414.

Dunn, M. J., Killcross, S. (2007b). Medial prefrontal cortex infusion of alpha-flupenthixol attenuates systemic d-amphetamine-induced disruption of conditional discrimination performance in rats. Psychopharmacology, 192, 347-55.

Feenstra, M. G. P. (2007). Microdialysis of dopamine and norepinephrine during conditioning and operant behaviour. In: B. H. C. Westerink \& T. I. F. H. Cremers (Eds.), Handbook of microdialysis: methods, applications and perspectives (pp317350). London: Academic Press.

Gold, J. M., Bish, J. A., Iannone, V. N., Hobart, M. P., Queern, C. A., \& Buchanan, R. W. (2000). Effects of contextual processing on visual conditional associative learning in schizophrenia. Biol. Psychiat., 48, 406-414.

Goldman-Rakic, P. S. (1991). Prefrontal cortical dysfunction in schizophrenia: The relevance of working memory. In B. J. Carroll \& J. E. Barrett (Eds.), Psychopathology and the Brain (pp 1-23). New York: Raven Press.

Guilera, G., Pino, O., Gómez-Benito, J., \& Rojo, J. E. (2009). Antipsychotic effects on cognition in schizophrenia: A meta-analysis of randomised controlled trials. Eur. J. Psychiat., 23, 77-89.

Guillin, O., Abi-Dargham, A., Laruelle, M., (2007). Neurobiology of dopamine in schizophrenia. Int. Rev. Neurobiol., 78, 1-39.

Heffner, T. G., Hartmanm, J. A., \& Seiden, L. S. (1980). Feeding increases dopamine metabolism in the rat brain. Science, 208, 1168-1170.

Hernandez, L., \& Hoebel, B. G. (1988). Feeding and hypothalamic stimulation increase dopamine turnover in the accumbens. Physiol. Behav., 44, 599-606.

Hernandez, L., \& Hoebel, B. G. (1990). Feeding can enhance dopamine turnover in the prefrontal cortex. Brain Res. Bull., 25, 975-979. 
Hofer, E., Doby, D., Anderer, P., \& Dantendorfer, K., (2001). Impaired conditional discrimination learning in schizophrenia. Schizophr. Res., 51, 127-136.

Izaki, Y., Hori, K., \& Nomura, M. (1998). Dopamine and acetylcholine elevation on leverpress acquisition in rat prefrontal cortex. Neurosci. Lett., 258, 33-36.

Jackson, M. E., Frost, A. S., \& Moghaddam, B. (2001). Stimulation of prefrontal cortex at physiologically relevant frequencies inhibits dopamine release in the nucleus accumbens. J. Neurochem., 78, 920-923.

Javitt, D. C., Shelley, A-M., Silipo, G., \& Lieberman, J. A. (2000). Deficits in auditory and visual context-dependent processing in schizophrenia: defining the pattern. Arch. Gen. Psychiat., 57, 1131-1137.

Jones, J. L., Day, J. J., Aragona, B. J., Wheeler, R. A., Whightman, R. M., \& Carelli, R. M. (2010). Basolateral amygdala modulates terminal dopamine release in the nucleus accumbens and conditioned responding. Biol. Psychiat., 67, 737-744.

King, D., Zigmond, M. J., \& Finlay, J. M. (1997). Effects of dopamine depletion in the medial prefrontal cortex on the stress-induced increase in extracellular dopamine in the nucleus accumbens core and shell. Neurosci., 77, 141-153.

Louilot, A., Le Moal, M., \& Simon, H. (1986). Differential reactivity of dopaminergic neurons in the nucleus accumbens in response to different behavioral situations. An in vivo voltammetric study in free moving rats. Brain Res., 397, 395-400.

Melis, M., Diana, M., \& Gessa, G. L. (1999). Clozapine potently stimulates mesocortical dopamine. Eur. J. Pharmacol., 366, R11-R13.

Merali, Z., Mclntosh, J., \& Anisman, H. (2004). Anticipatory cues differentially provoke in vivo peptidergic and monoaminergic release at the medial prefrontal cortex. Neuropsychopharmacology, 29, 1409-1418.

Moghaddam, B., Bunney, B.S. (1990). Acute effect of typical and atypical neuroleptics on the release of dopamine from prefrontal cortex, nucleus accumbens, and striatum of the rat: An in vivo microdialysis study. J. Neurochem., 54, 1755-1760.

Neill, D. B., Fenton, H., \& Justice, J. B. Jr (2002). Increase in accumbal dopaminergic transmission correlates with response cost not reward of hypothalamic stimulation. Behav. Brain Res., 137, 129-138. 
Nomura, M., Izaki, Y., Takita, M., Tanaka, J., \& Hori, K. (2004). Extracellular level of basolateral amygdala dopamine responding to reversal of appetitive-conditioned discrimination in young and old rats. Brain Res., 1018, 241-246.

Paxinos, G., \& Watson, C. (2004). The Rat Brain in Stereotaxic Coordinates. New York: Elsevier.

Petrides, M. (1985). Deficits on conditional associative-learning tasks after frontal- and temporal-lobe lesions in man. Neuropsychologia, 23, 601-614.

Petrides, M. (1990). Nonspatial conditional learning impaired in patients with unilateral frontal but not unilateral temporal lobe excisions. Neuropsychologia, 28, 137-149.

Petrides, M. (1997). Visuo-motor conditional associative learning after frontal and temporal lesions in the human brain. Neuropsychologia, 35, 989-997.

Phillips, A. G., Atkinson, L. J., Blackburn, J. R., \& Blaha, C. D. (1993). Increased extracellular dopamine in the nucleus accumbens of the rat elicited by a conditioned stimulus for food: an electrochemical study. Can. J. Physiol. Pharamcol., 71, 387393.

Phillips, A. G., Ahn, S., \& Floresco, S. B. (2004). Magnitude of dopamine release in medial prefrontal cortex predicts accuracy of memory on a delayed response task. J. Neurosci., 24, 547-553.

Robbins, T. W., \& Sahakian, B. J. (1983). Behavioural effects of psychomotor stimulant drugs: clinical and neuropsychological implications. In: I. Creese (Ed.) Stimulants: neurochemical behavioural and clinical perspectives ( $\mathrm{pp}$ 301-338). New York: Raven.

Rossetti, Z. L., \& Carboni, S. (2005). Noradrenaline and dopamine elevations in the rat prefrontal cortex in spatial working memory. J. Neurosci., 25, 2322-2329.

Santiago, M., \& Westerink, B. H. C. (1990). Characterization of the vivo release of dopamine as recorded by different types of intracerebral microdialysis probes. Naunyn-Schmiedeberg's Arch. Pharmacol., 342, 407-414.

Sesack, S. R., \& Carr, D. B. (2002). Selective prefrontal cortex inputs to dopamine cells: implications for schizophrenia. Physiol. Behav., 77, 513-517. 
Sokolowski, J. D., Conlan, A. N., \& Salamone, J. D. (1998) A microdialysis study of nucleus accumbens core and shell dopamine during operant responding in the rat. Neuroscience, 86, 1001-1009.

Stefani, M. R., \& Moghaddam, B. (2006). Rule learning and reward contingency are associated with dissociable patterns of dopamine activation in the rat prefrontal cortex, nucleus accumbens, and dorsal striatum. J. Neurosci., 26, 8810-8818.

Stuber, G. D., Klanker, M., de Ridder, B., Bowers, M. S., Joosten, R. N., Feenstra, M. G., \& Bonci, A. (2008). Reward-predictive cues enhance excitatory synaptic strength onto midbrain dopamine neurons. Science, 321, 1690-1692.

Sunsay, C., \& Rebec, G. V. (2008). Real-time dopamine efflux in the nucleus accumbens core during Pavlovian conditioning. Behav. Neurosci., 122, 358-367.

Umbricht, D., Schmid, L., Koller, R., Vollenweider, F. X., Hell, D., \& Javitt, D. C. (2001). Ketamine-induced deficits in auditory and visual context-dependent processing in healthy volunteers: implications for models of cognitive deficits in schizophrenia. Arch. Gen. Psychiat., 57, 1139-1147.

Weinberger, D. R., Aloia, M. S., Goldberg, T. E., \& Berman, K. F. (1994). The frontal lobes and schizophrenia. J. Neuropsychiatry Clin. Neurosci. 6, 419-427.

Weinberger, D., and Laruelle, M. (2001). Neurochemical and neuropharmachological imaging in schizophrenia. In K. L. Davis, D. S. Charney, J. T. Coyle, \& Charles Nemeroff (Eds.), Neuropsychopharmacology: The Fifth Generation of Progress (pp. 833-856). Lippincott, Williams, and Wilkins. New York.

Wilkinson, L. S., Humby, T., Killcross, A. S., Everitt, B. J., \& Robbins, T. W. (1998). Dissociations in dopamine release in medial prefrontal cortex and ventral striatum during the acquisition and extinction of classical aversive conditioning in the rat. Eur. J. Neurosci., 10, 1019-1026.

Wysocki, M. S., \& Sweet, J. J. (1985). Identification of brain-damaged schizophrenic, and normal medical patients using a brief neuropsychological screening battery. Int. J. Clin. Neuropsy., 7, 40-49. 
Xiang, J.-Z., \& Brown, M. W. (2007). Comparison of differential neuronal responsiveness for different regions of the prefrontal cortex in a conditional visual discrimination task. Eur. J. Neurosci., 25, 2916-2926.

Youngren, K. D., Inglis, F. M., Pivirotto, P. J., Jedema, H. P., Bradberry, C. W., GoldmanRakic, P. S., Roth, R. H., \& Moghaddam, B. (1999). Clozapine preferentially increases dopamine release in the rhesus monkey prefrontal cortex compared with the caudate nucleus. Neuropsychpharmacology, 20, 403-412. 


\section{Abbreviations}

DA - dopamine

DAergic - dopaminergic

mPFC - medial prefrontal cortex

$\mathrm{NAc}$ - nucleus accumbens 
Table 1

Simplified design of the experiments

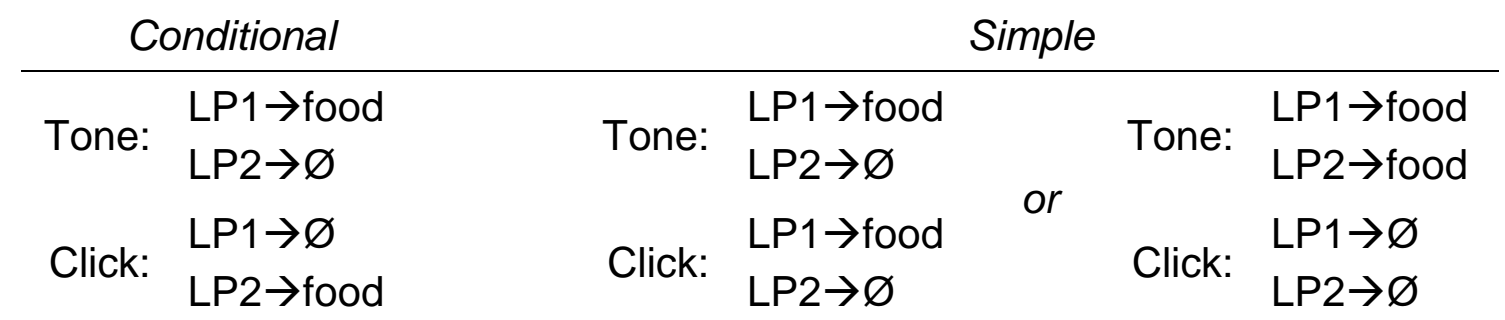

Note: The stimuli were a $2 \mathrm{kHz}$ tone and a $10 \mathrm{~Hz}$ train of clicks. Two response levers, LP1 and LP2 were available throughout the experiment. Where appropriate, responding on the levers was reinforced by the delivery of food pellets according to a random interval 15-s schedule. At other times responses were not reinforced $(\varnothing)$. For rats learning the conditional discrimination, responses on LP1 were reinforced in the presence of the tone, but not in the presence on the clicker, whereas responses on LP2 were reinforced in the presence of the clicker but not the tone. For some rats learning the simple discrimination, responses on one of the levers (LP1) were reinforced in the presence of either stimulus whereas responses on the alternative lever (LP2) were never reinforced. For the remaining simple discrimination animals, responses on either of the levers were reinforced in the presence of one of the auditory stimuli, but not in the presence of the other. 


\section{Figure Legends}

Figure 1. Drawings of coronal sections showing the placement of the $2 \mathrm{~mm}$ dialysis membrane of microdialysis probes in medial prefrontal cortex and nucleus accumbens for rats in Experiment 1 (A), and Experiment 2 (B). The numbers (in $\mathrm{mm}$ relative to bregma) denote the anterior-posterior level of the illustrated sections in correspondence to the stereotaxic rat brain atlas by Paxinos and Watson (1998).

Figure 2. The mean rates of correct (+) and incorrect (-) lever-press responding during the initial $10 \mathrm{~s}$ of each presentation of the discriminative stimuli for animals learning either the conditional or the simple discrimination over the 8 sessions of training, the post-operative retraining session, and the test session of Experiment 1 (A), and Experiment 2 (B). All animals learned to respond more on the correct than the incorrect lever. There were no significant differences in the performance of animals in the conditional and simple discrimination groups on either the retraining or test session of each experiment. Error bars show one standard error of the mean.

Figure 3. Dopamine concentrations in the dialysate collected from the medial prefrontal cortex in Experiment $1(A)$, and the nucleus accumbens in Experiment $2(B)$, expressed as a percentage of basal dopamine levels. Samples were collected every 10 mins during a 60 min baseline period, a 60 min behavioural test session, and a 60 min recovery period that followed the test session. The hatched bar indicates the time course of the behavioural test session. Histograms show the averages for the six samples collected during the test session and the six samples collected during the recovery period for animals learning each of the two discriminations. In Experiment 1 levels of prefrontal cortex DA were greater for rats performing the conditional discrimination than those performing the simple discrimination during the recovery period. Error bars show one standard error of the mean. In Experiment 2, the simple discrimination was associated with higher levels of dopamine (DA) efflux in the nucleus accumbens across the behavioural test session and recovery period than the conditional discrimination. 
Figure 1

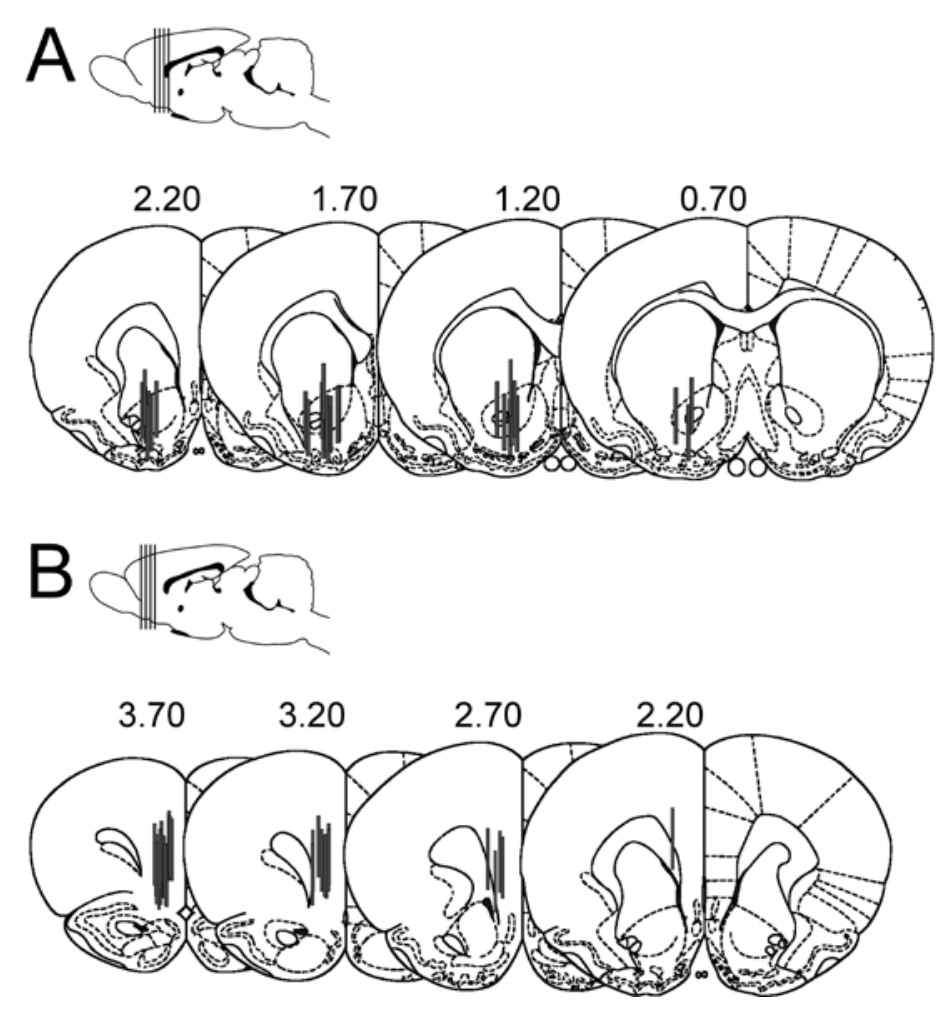


Figure 2

A
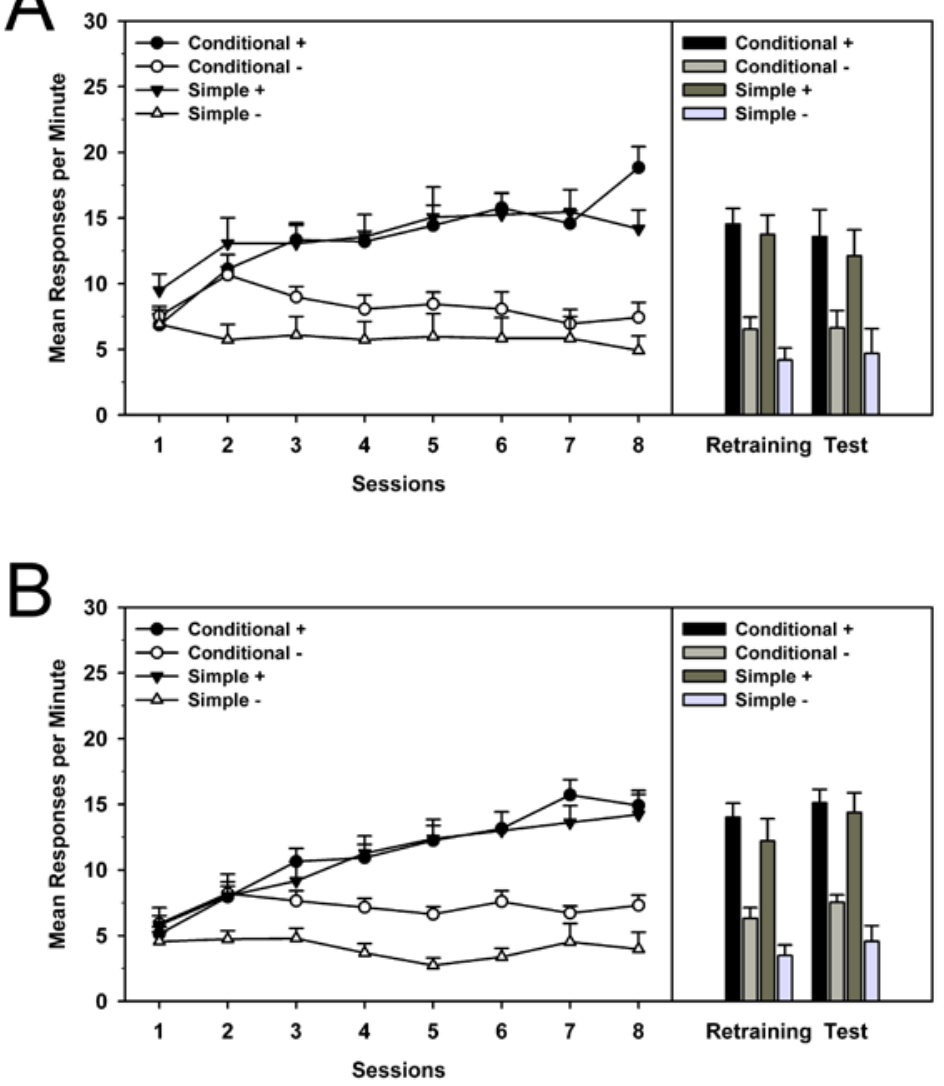
Figure 3
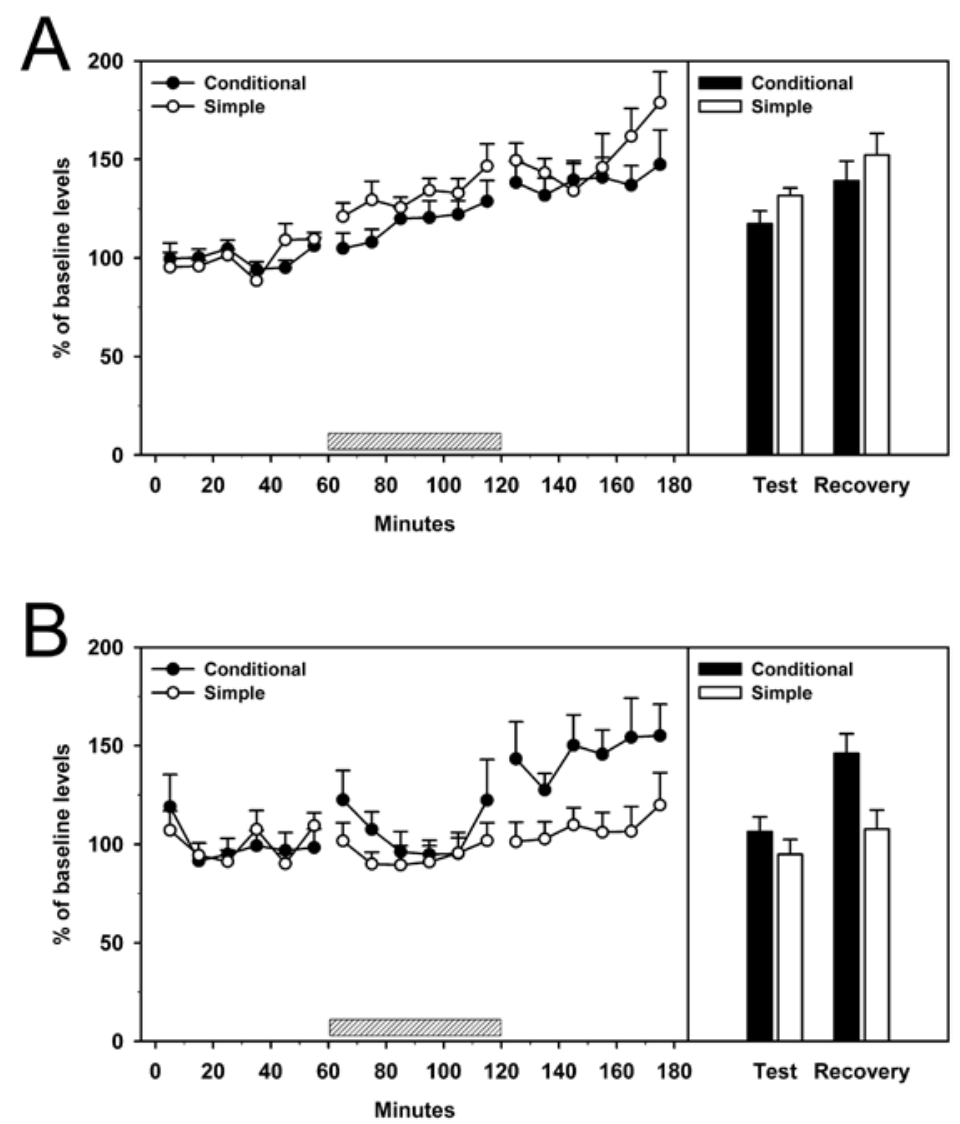